\title{
Statistical Study of Zig-Zag Transition Boundaries in Longitudinal Digital Magnetic Recording
}

\author{
Aleksandar Kavčić, Student Member, IEEE, and José M. F. Moura, Fellow, IEEE
}

\begin{abstract}
In this paper, we study the statistics of zig-zag transition walls in digital magnetic recording and their relationship to transition noise defining quantities. We provide analytic results that link the statistics of zig-zag transitions to media/recording parameters. The basis of our study is the triangle zig-zag transition (TZ-ZT) model due to its well-defined triangle zig-zag shape and its cross-track stability. The results we derive here, however, are of a general nature, and given the right interpretation, apply to other zig-zag models as well, as we show in the paper. We also provide an interpretation of the cross-track correlation width, linking this quantity to the statistics of magnetized clusters in thin-film magnetic media. The paper concludes by showing how these results can be used in media noise modeling.
\end{abstract}

\section{INTRODUCTION}

$\mathbf{I}$ T HAS LONG been observed that most of the noise in thin film magnetic recording media occurs in the transitional regions between two oppositely magnetized magnetization patterns in the media [1], [2]. The source of this noise lies in the irregularity of the transition boundary. The zig-zag boundary itself is a realization of a random process, which is why the observed media noise is indeed noise, i.e., random.

In the past, there have been many studies of zig-zag wall shapes. Several zig-zag models have been suggested. These models cluster in two groups: deterministic and stochastic models. Examples of deterministic models are described in [3]-[6]. While deterministic models are useful in understanding the physical mechanisms involved in the recording process, they cannot explain the random character of noise. The stochastic models [7]-[11] model the zig-zag transition line as a random process, thus accounting for the random character of media noise.

Widely accepted models for describing the physics of thinfilm magnetic media are the micromagnetic models [12]-[14]. While micromagnetic models model the fine microphysics of each particle (grain) of the recording media, stochastic zig-zag models rely on extracting only a few random parameters from macroscopic measurements and extrinsic media properties to accurately model the transitions and transition noise. The tradeoff between the micromagnetic models and stochastic zigzag models is between accuracy and speed. The stochastic zigzag models are much faster, whereas micromagnetic models provide greater detail. In detection studies, fast models, like

Manuscript received September 13, 1996; revised June 4, 1997. This work was supported in part by the National Science Foundation under Grant ECD8907068. The United States government has certain rights in this material.

The authors are with the Data Storage Systems Center, Department of Electrical and Computer Engineering, Carnegie Mellon University, Pittsburgh, PA 15213 USA.

Publisher Item Identifier S 0018-9464(97)06894-5. the stochastic zig-zag models we describe in this paper, are necessary in order to generate the large number of transitions required to provide statistical significance to the error rate computations.

In this paper, we present a statistical study of zig-zag transition walls in longitudinal magnetic recording. Rather than analyzing the statistics of the zig-zag walls, we are more concerned with the synthesis. Synthesis involves choosing the parameters in the random zig-zag process such that the process is stable and such that these parameters can be related to macroscopic (measurable) media/recording properties. The triangle zig-zag transition (TZ-ZT) model that we propose is stable. We show how to relate its parameters to the media physical characteristics. Although the derivation of our theoretical results is tied to the TZ-ZT model, the results are general and can be applied to other similar models. In particular, we mention how to modify the model in [9], [10] to exhibit cross track stability. We also derive an alternative expression for the cross-track correlation width and show that it applies not only to the TZ-ZT model but to such square-wave type models as the microtrack model in [15] and [16].

This paper is focused on the presentation of theoretical results for isolated transitions. Coupling these results for isolated transitions with models for bit shift, transition position variance increase [15], [17], and percolation thresholds [16], [18], we can easily extend our TZ-ZT model to include the nonlinear effects of interacting transitions [19].

The paper is organized as follows. Section II reviews briefly the TZ-ZT model as well as other proposed stochastic zigzag transition models. Section III derives novel analytic results linking the statistics of zig-zag patterns to measurable media/recording quantities. While Section III is kept short, the proofs, discussions, and examples supporting the results derived in Section III are in the Appendix. Section IV gives an example of how the results derived in Section III can be used to synthesize zig-zag transitions. Section V concludes the paper.

\section{TRIAngle Zig-Zag Transition Modeling}

The use of stochastic zig-zag wall models as media noise models in magnetic recording has first been suggested by Arnoldussen and Tong [7]. Based on Lorentz-microscopy pictures of zig-zag transitions in the media, they extracted the probability density functions (pdf's) of the sawtooth lengths (which are denoted by $w_{1}, w_{2}, \cdots$ in Fig. 1) and of the zigzag vertex angles (which are denoted by $\theta_{1}, \theta_{2}, \cdots$ in Fig. 1 ). While obtaining the pdf's of the sawtooth lengths $w_{i}$ and of 


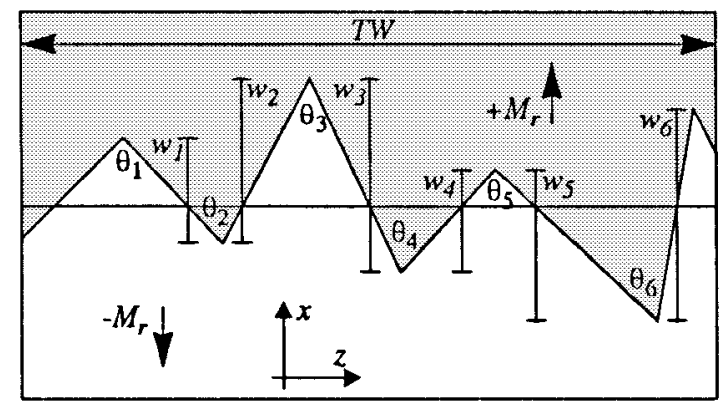

Fig. 1. Stochastic zig-zag transition model. Labeled are the sawtooth lengths and vertex angles.

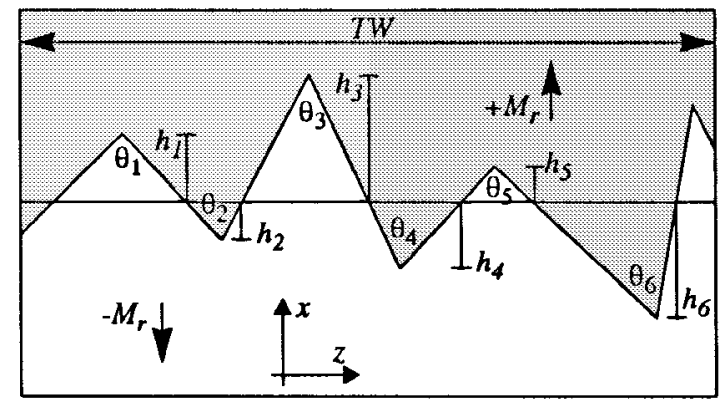

Fig. 2. Stochastic zig-zag transition model. Labeled are zero-to-peak distances and vertex angles.

the vertex angles $\theta_{i}$ suffices for the analysis of the zig-zag patterns, it does not provide a good tool for their synthesis. The main reason is the instability of the model in [7]. If the sawtooth lengths $w_{1}, w_{2}, \cdots$ are used as independent random variables in the synthesis process, the resulting stochastic zig-zag process is an independent increment random process [11], [20]. The variance of an independent increment random process grows with time (in this case with the cross-track distance). This makes the synthesized zig-zag process unstable with a drift in the down-track direction due to the growth of the variance with the cross-track distance. This problem has been recognized in a follow-up paper by Tang and Osse [8], who suggest deconvolving the pdf of the sawtooth lengths $w_{i}$ to obtain the pdf of the zero-to-peak lengths (denoted by $h_{1}, h_{2}, \cdots$ in Fig. 2). Using the zero-to-peak distances as independent random variables stabilizes the model. Although stable, the model in [8] was impractical to use since it relied on Lorentz microscopy pictures for the determination of the pdf's used. Obtaining the photographs of the transitions in the media is a lengthy process.

A more systematic approach was taken by Middleton and Miles in their model described in [9] and [10]. These authors assume the vertex angle $\theta$ to be constant for all zig-zags and derive a relationship between the pdf of the sawtooth lengths $w_{1}, w_{2}, \cdots$ and the media hysteresis loop. There are several problems that remain with this model as well. First, the question of how to choose the vertex angle $\theta$ remained unanswered. Second, the distinction between centered and noncentered sawtooth lengths has not been made, and both were inconsistently used in the figures and equations of [9] and [10], which leads to possible instability of the model. Third,

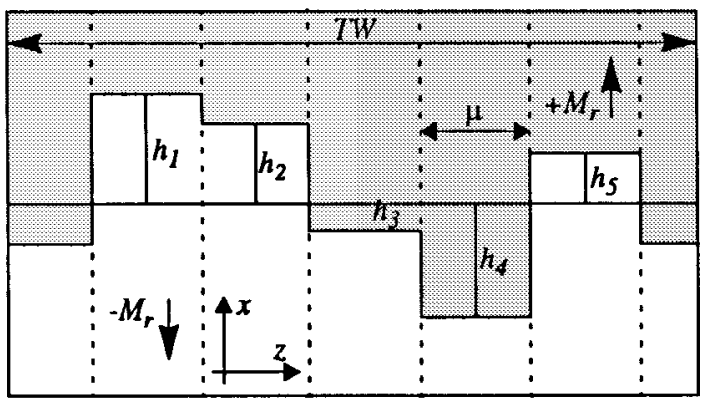

Fig. 3. Microtrack model. The microtrack width is denoted with $\mu$.

and most important, the paradox of residual life in renewal theory [21], [22] was not recognized, which misled the authors into deriving an incorrect relationship between the sawtooth lengths pdf and the magnetization profile.

Perhaps these hardships in dealing analytically with stochastic zig-zag models led to the popularity of such simpler stochastic square wave type models as the microtrack model [15], [16]. In the microtrack model (see Fig. 3), the track is divided into microtracks. The magnetization reversal in each microtrack is a perfect step function, where the exact position of the reversal is governed by the pdf of the square wave heights (which is denoted by $h_{1}, h_{2}, \cdots$ in Fig. 3). It is fairly easy to show that the pdf of the heights $h_{i}$ in the microtrack model in Fig. 3 equals the normalized first derivative of the magnetization profile, whereas the microtrack width equals the cross-track correlation width of the model. At the same time, this answers the question of how to set the pdf of the $h_{i}$ 's and the microtrack width, which makes the model easy to use.

Our goal in this paper is to derive relationships that will make the stochastic zig-zag transition models just as easy to use as the microtrack model. We derive formulas that link the statistics of a zig-zag transition to the magnetization profile and to the cross-track correlation width. However, before we do that, we need a stochastic zig-zag model that is both stable in the cross-track direction (nonindependent increment random process) and is analytically tractable. This model is the TZ-ZT model [11], [17], which we describe briefly next.

\section{A. Triangle Zig-Zag Transition Model}

The TZ-ZT model is depicted in Fig. 4. The magnetization of the media is allowed to be oriented only in the downtrack direction, with a value $\pm M_{r}$, where $M_{r}$ is the remanent magnetization. The regions of oppositely oriented magnetizations are separated by a zig-zag line centered at the nominal transition location. The zig-zag line is determined by the heights of the vertices $h_{i}$ and the vertex angle $\theta$. We choose $\theta$ to be a constant, although a more complex model could treat $\theta$ as a random variable. The constant angle $\theta$, however, makes the model analytically tractable, which allows us to derive results in Section III. The heights $h_{i}$ are chosen to be samples of independent, identically distributed (iid) random variables. Thus, the zig-zag line is obtained by truncating isosceles triangles of heights $h_{i}$ on the same basis line, which is the line perpendicular to the recording direction at the nominal transition location. The truncated triangles are of alternating 


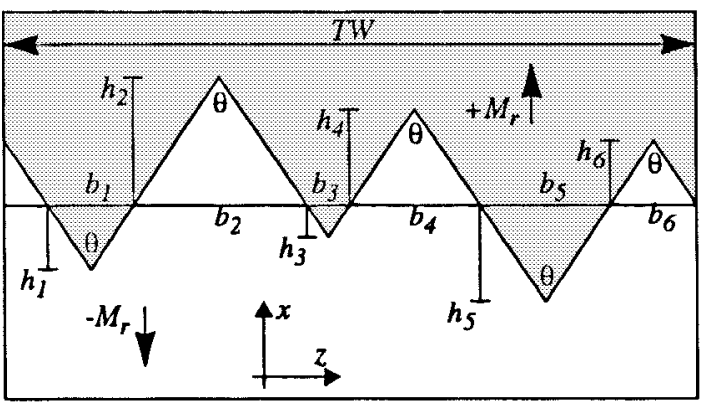

Fig. 4. Triangle zig-zag transition model.

orientation, one pointing up, the next one down, then up again, and so on, until the whole track width has been swept. We refer to the model as the TZ-ZT model. Notice that the independent random variable in this model is the triangle height and not the sawtooth length. If we start drawing a triangle at the basis line, we would go $h_{i}$ in one direction and then come back by the same length $h_{i}$ to finish at the nominal transition location. This ensures that the zig-zag line does not drift away from the nominal transition location. Therefore, unlike independent increment random processes, the TZ-ZT model is stable.

\section{STATISTICAL RELATIONSHIPS}

As described earlier, the inputs for the TZ-ZT model (which are restricted here only to isolated transitions) are the pdf of the triangle heights $f_{H}(h)$ and the vertex angle $\theta$. In the following subsections we show the relationships between these quantities and the recording process parameters. In particular, we show that the pdf $f_{H}(h)$ is related to the transition profile and that the vertex angle $\theta$ is determined by the cross-track correlation width of the modeled thin film medium.

\section{A. Triangle Heights pdf $f_{H}(h)$}

This subsection links the statistics of the zig-zag triangles to the magnetization profile of an isolated transition. Assume that we have an infinitely wide track, across which we have a zig-zag transition generated by the TZ-ZT model; see Fig. 4 for a picture of the TZ-ZT model. Let $f_{H}(h)$ be the pdf of the triangle heights, and let $M(x)$ be the magnetization transition profile of an isolated transition. We assume that $M(x)$ is an odd function, for example, $M(x)=M_{r}(2 / \pi) \operatorname{atan}(x / a)$ or $M(x)=M_{r} \operatorname{erf}(x / \sqrt{2} \sigma)$. By $M_{r}$, we denote the remanent magnetization. Let $T W$ denote the track width. Without loss of generality, we will assume in the remainder of this subsection that $M_{r}=1$.

Theorem 1: The magnetization profile $M(x)$ of an isolated transition created by the TZ-ZT model on an infinitely wide track $(T W \rightarrow \infty)$ is related to the triangle heights pdf $f_{H}(h)$ through

$$
f_{H}(h)= \begin{cases}-\frac{M^{\prime \prime}(h)}{M^{\prime}(0)} & \text { for } h \geq 0 \\ 0 & \text { for } h<0\end{cases}
$$

where $M^{\prime}(0)$ denotes the first derivative of $M(x)$ at $x=0$ (i.e., the slope of the transition profile), and $M^{\prime \prime}(x)$ is the second derivative of the transition profile.
The proof is presented in the Appendix.

Theorem 1 sets the triangle heights pdf $f_{H}(h)$ of the TZZT model to the desired magnetization profile of an isolated transition $M(x)$.

In [9] and [10], a relationship was derived between the magnetization profile and the statistics of the saw-tooth zigzag model (see [10, eq. (5)]). This equation is not correct since it neglects the residual life paradox of renewal theory [21], see the Appendix for details. In [9] and [10], the distinction between centered and noncentered saw-tooth lengths $W$ is not made either, making the model potentially unstable, depending on the interpretation. If we modify the model in [10] to account for the residual life paradox and centered saw-tooth lengths, we find that the true relationship linking the magnetization profile to the saw-tooth pdf is very similar to (1). From this pdf, it is easy to derive the simple relationship between the triangle heights $H$ and the saw-tooth lengths $W, W=2 H$.

\section{B. Cross-Track Correlation Width and the Vertex Angle $\theta$}

The size of the vertex angle $\theta$ will determine how much jitter there is in the readback signal, i.e., how much jitter in the readback signal is a result of the zig-zag transitions in the media. If the angle $\theta$ is small, that means that many zig-zags occur across the track width. Since the head senses the average of all the zig-zags across the track, jitter is not strong. If the angle $\theta$ is large, however, fewer zig-zags will occur across the track width, fewer zig-zags are therefore averaged across the track, and more jitter is present. This discussion shows that it is reasonable to make the vertex angle $\theta$ dependent on a quantity directly tied to the amount of jitter media noise. This quantity is the cross-track correlation width [15].

Let a magnetization transition be written at $x=0$. The magnetization associated with that transition is a two dimensional (2-D) random process $m(x, z)$, where $x$ is the down-track direction, and $z$ is the cross-track direction. Let $\mathrm{E}$ denote the expectation operator (statistical average operator). Then, the normalized cross-track magnetization autocorrelation function $r(Z)$ at $x=0$ is defined as [15]

$$
\begin{aligned}
& r(Z)= \\
& \frac{\mathrm{E}[(m(0, z)-\mathrm{E}[m(0, z)])(m(0, z+Z)-\mathrm{E}[m(0, z+Z)])]}{M_{r}^{2}}
\end{aligned}
$$

where $Z$ is the cross-track displacement. We are assuming that the magnetization process $m(x, z)$ is stationary in the crosstrack direction, which implies that the average magnetization profile

$$
M(x)=\mathrm{E}[m(x, z)]
$$

is independent of $z$. In addition, since we assumed that the transition is written at $x=0$, we have $M(0)=\mathrm{E}[m(0, z)]=$ 0 . Therefore, (2) simplifies to

$$
r(Z)=\frac{\mathrm{E}[m(0, z) m(0, z+Z)]}{M_{r}^{2}} .
$$


The cross-track correlation width $s$ is defined as the area under the cross track correlation function

$$
s=\int_{Z=-\infty}^{\infty} r(Z) d Z
$$

We now find an expression for the cross-track correlation width of the TZ-ZT model.

Theorem 2: The cross-track correlation width $s_{\mathrm{TZ}-\mathrm{ZT}}$ of the TZ-ZT model is related to the statistics of the triangle bases $B$ of the TZ-ZT model through

$$
s_{\mathrm{TZ}-\mathrm{ZT}}=\frac{\operatorname{Var}(B)}{\mathrm{E}[B]}
$$

where $\operatorname{Var}(B)$ stands for the variance of the random triangle base $B$, and $\mathrm{E}(B)$ stands for the expected value of $B$.

The proof of Theorem 2 is in the Appendix.

Although Theorem 2 is derived in the context of the TZ-ZT model, its proof holds in a fairly general context. The theorem gives an alternative interpretation of the cross-track correlation width. Rewrite the equation in Theorem 2 in its general form

$$
s=\frac{\operatorname{Var}(C)}{\mathrm{E}[C]} .
$$

Here, $s$ is the cross-track correlation width, and $C$ is a random variable representing the cross-track size of a magnetization cluster in the dc demagnetized state of the medium. It is assumed that the magnetization of each cluster is either $+M_{r}$ or $-M_{r}$ pointing along the recording direction. For a better understanding of what we mean by magnetization clusters, refer to Fig. 5, where a portion of a dc demagnetized medium is shown in a simplified sketch. Note that clusters are not the same as grains (there might be more than one grain per cluster). Equation (7) gives the relationship between the statistics of magnetization cluster sizes and the cross-track correlation width. It clearly shows that the cross-track correlation width is not the average cluster size, and it should not be confused with the zig-zag half-wavelength (e.g., see [5] for the definition of the zig-zag wavelength). Equation (7) says that the crosstrack correlation width is the ratio between the variance and the mean of the cluster sizes. Thus, a low noise (low crosstrack correlation width) medium has the cluster size variance $\operatorname{Var}(C)$ that is small compared with the cluster mean $\mathrm{E}[C]$. In other words, it is not the average cluster size but the regularity of the cluster sizes (relative to the average size) that determines the level of the media noise.

To verify (7), we next apply it to an alternative media noise model [the microtrack media model [15], [16] (Fig. 3)], to show that (7) correctly equates the cross-track correlation width to the microtrack width. Of course, for the microtrack model, the cross-track correlation width can be easily calculated using the integral definition (5). The objective here, however, is to demonstrate the validity of (7) in an example involving a model different from the TZ-ZT model.

To use (7), we need to determine the mean and variance of the cross-track cluster sizes $C$ for the microtrack model; see Fig. 3 for a picture of the microtrack model. From Fig. 3 we see that the cross-track magnetization cluster size can only take values $C=i \mu$, where $i$ is a positive integer, and $\mu$ is

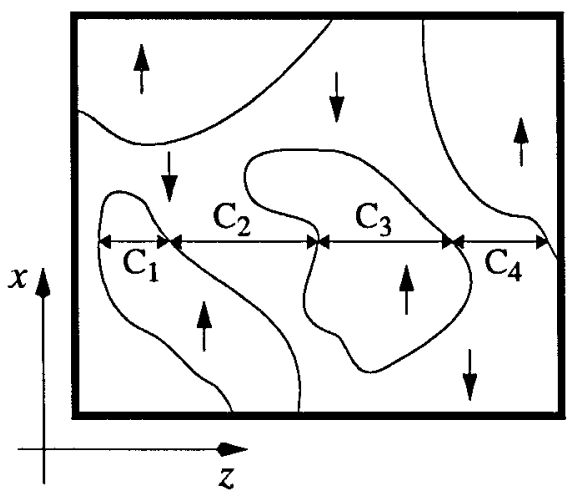

Fig. 5. Portion of a de demagnetized medium showing magnetization clusters. The magnetization of each cluster is assumed to be parallel to the recording direction with value either $+M_{r}$ or $-M_{r}$. The cross-track dimensions of a few clusters are denoted with $C_{i}, i=1, \cdots, 4$.

the microtrack width. Since the transition position (which is denoted by $h_{i}$ in Fig. 3) for each microtrack is independent of the transition position in any other microtrack, we have that the probability that the cluster size equals $i \mu$ is

$$
\mathrm{P}\{C=i \mu\}=\frac{1}{2^{i}}, \quad \text { for } \quad i=1,2, \cdots .
$$

Having the probability measure in (8), we calculate the mean cluster size $\mathrm{E}[C]$, the mean square cluster size $\mathrm{E}\left[C^{2}\right]$, and the cluster size variance $\operatorname{Var}(C)=\mathrm{E}\left[C^{2}\right]-\mathrm{E}[C]^{2}$ as

$$
\begin{aligned}
\mathrm{E}[C] & =\sum_{i=1}^{\infty} i \mu \mathrm{P}\{C=i \mu\}=\sum_{i=1}^{\infty} i \mu \frac{1}{2^{i}}=2 \mu \\
\mathrm{E}\left[C^{2}\right] & =\sum_{i=1}^{\infty}(i \mu)^{2} \mathrm{P}\{C=i \mu\}=\sum_{i=1}^{\infty}(i \mu)^{2} \frac{1}{2^{i}}=6 \mu^{2} \\
\operatorname{Var}(C) & =\mathrm{E}\left[C^{2}\right]-\mathrm{E}[C]^{2}=6 \mu^{2}-(2 \mu)^{2}=2 \mu^{2} .
\end{aligned}
$$

Substituting (9) and (11) into (7), we get the cross-track correlation width

$$
s=\frac{\operatorname{Var}(C)}{\mathrm{E}[C]}=\frac{2 \mu^{2}}{2 \mu}=\mu
$$

i.e., the cross-track correlation width of the microtrack model equals the microtrack width $\mu$.

Given the result in Theorem 2, we find now an expression for the vertex angle $\theta$ such that the cross-track correlation width of the TZ-ZT model equals the cross-track correlation width of the media we are modeling.

Corollary 2.1: The TZ-ZT modeled medium and the thin film medium being modeled have the same cross-track correlation width $s$ if the TZ-ZT vertex angle $\theta$ is

$$
\theta=2 a \tan \left[\frac{s \cdot \mathrm{E}[H]}{2 \cdot \operatorname{Var}(H)}\right]
$$

where $H$ is the random variable representing the TZ-ZT heights.

Proof 2.1: The bases $b_{i}$ are related to the heights $h_{i}$ as $b_{i}=2 h_{i} \cdot \tan (\theta / 2)$, see Fig. 4. If we substitute $B=$ $H \cdot 2 \tan (\theta / 2)$ into (6) and solve the resulting equation for $\theta$, we get (13). 


\section{Matching the TZ-ZT Model to Physical Media}

We demonstrate through an example how the TZ-ZT defining quantities (the triangle heights pdf and the vertex angle) are obtained from measurements of magnetization profiles of isolated transitions. We use transition profiles obtained by micromagnetic modeling. The same information can be obtained from spin-stand measurements by deconvolving the head transfer function from the isolated pulse shapes [19].

Here, we demonstrate the extraction procedure for two types of thin film media. The first one is an oriented medium with the orientation ratio O.R. $=1.3$, The second one is an isotropic medium with O.R. $=1.0$.

\section{A. Oriented Thin-Film Medium}

Using the micromagnetic model of [14], we simulated a thin film magnetic medium with the following characteristics: media thickness $\delta=250 \AA$; coercivity $H c=1770$ Oe; remanence $M_{r}=600 \mathrm{emu} / \mathrm{cm}^{3}$; orientation ratio O.R. $=1.3$. We used a Karlquist write head with the following geometry: magnetic spacing (flying height) $d=0.1 \mu \mathrm{m}$; gap length $g=$ $0.24 \mu \mathrm{m}$; track width $T W=1.25 \mu \mathrm{m}$. With this head-media geometry, we wrote 500 independent transitions $M_{i}(x), i=$ $1, \cdots, 500$ and found the average transition profile

$$
M(x)=\frac{1}{500} \sum_{i=1}^{500} M_{i}(x) .
$$

We could use this averaged magnetization profile $M(x)$ directly in Theorem 1 to obtain the pdf $f_{H}(h)$. Instead, we first find an analytic fit to $M(x)$

$$
\frac{M(x)}{M_{r}} \approx M_{a}(x)=\operatorname{erf}\left(\frac{x}{\sigma \sqrt{2}}\right) .
$$

The best fit in a least squares sense leads to $\sigma=460 \AA$.

With the analytic expression in (15), and using Theorem 1, we get the triangle heights pdf

$$
f_{H}(h)=-\frac{M_{a}^{\prime \prime}(h)}{M_{a}^{\prime}(0)}= \begin{cases}\frac{h}{\sigma^{2}} \exp \left(-\frac{h^{2}}{2 \sigma^{2}}\right) & h \geq 0 \\ 0 & h<0\end{cases}
$$

which is the well-known Rayleigh pdf. The mean and variance of a Rayleigh-distributed random variable are

$$
\mathrm{E}[H]=\sqrt{\frac{\pi}{2}} \sigma \quad \text { and } \quad \operatorname{Var}(H)=\left(2-\frac{\pi}{2}\right) \sigma^{2} .
$$

To find the cross-track correlation width $s$, we use the following relationship between the magnetization variance $\sigma_{M}(x)$ and the magnetization profile $M(x)$ [23].

$$
\sigma_{M}^{2}(x)=\frac{s}{T W}\left[1-\frac{M^{2}(x)}{M_{r}^{2}}\right] .
$$

The magnetization variance is defined as

$$
\begin{aligned}
\sigma_{M}^{2}(x) & =\frac{1}{M_{r}^{2}} \mathrm{E}\left[(m(x)-\mathrm{E}[m(x)])^{2}\right] \\
& =\frac{1}{M_{r}^{2}} \mathrm{E}\left[(m(x)-M(x))^{2}\right] .
\end{aligned}
$$

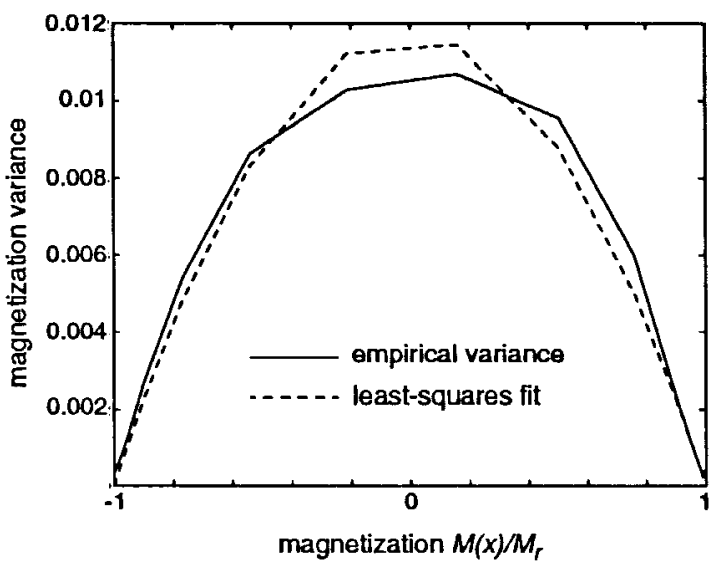

Fig. 6. Magnetization variance for the oriented medium. Solid line: empirical magnetization variance $\hat{\sigma}_{M}(x)$ versus the magnetization $M(x) / M_{r}$. Dotted line: Least-squares parabola fit.

We obtain the empirical magnetization variance $\hat{\sigma}_{M}^{2}(x)$ from measurements as

$$
\hat{\sigma}_{M}^{2}(x)=\frac{1}{499} \sum_{i=1}^{500}\left[M_{i}(x)-M(x)\right]^{2} .
$$

The plot of $\hat{\sigma}_{M}^{2}$ versus $M(x)$ is given in Fig. 6 as the solid line. The dotted line in Fig. 6 is obtained by least-squares fitting a parabola of the form in (18)-(20). The value for the cross-track correlation width that provides the fit is

$$
s=146.86 \AA \text {. }
$$

Substituting (21) and (17) into (13) of Corollary 2.1, with $\sigma$ $=460 \AA$, we obtain the vertex angle

$$
\theta=2 a \tan \left[\frac{s \cdot \mathrm{E}[H]}{2 \cdot \operatorname{Var}(H)}\right] \approx 50^{\circ} .
$$

With the triangle heights pdf in (16) and the vertex angle in (22), we generated 50000 independent transitions with the TZ-ZT model. We next make a statistical comparison between the transitions created by the micromagnetic model and those created with the TZ-ZT model. Fig. 7 shows a very good agreement between the average transition profiles of the micromagnetic model and the TZ-ZT model. In Fig. 8, we plotted the normalized histograms (empirical pdf's) of the position jitter for the micromagnetic model and the TZ-ZT model. Here we see a very good agreement as well. Although the TZZT model provides good matches for the average transition profile and jitter noise, we see the limitations of the TZ-ZT model in Fig. 9, where we plotted the normalized histograms of the transition slopes. The TZ-ZT model underestimates the variance of the transition slopes by about $30 \%$. We believe that the reason for this is that TZ-ZT models a perfect-step transition across the zig-zag boundary, whereas in real media the magnetization rotates across the wall with some finite wall width.

\section{B. Isotropic Thin-Film Medium}

We simulated an isotropic medium $(\mathrm{O} . \mathrm{R} .=1.0)$ with the following characteristics (same as for the oriented medium): 


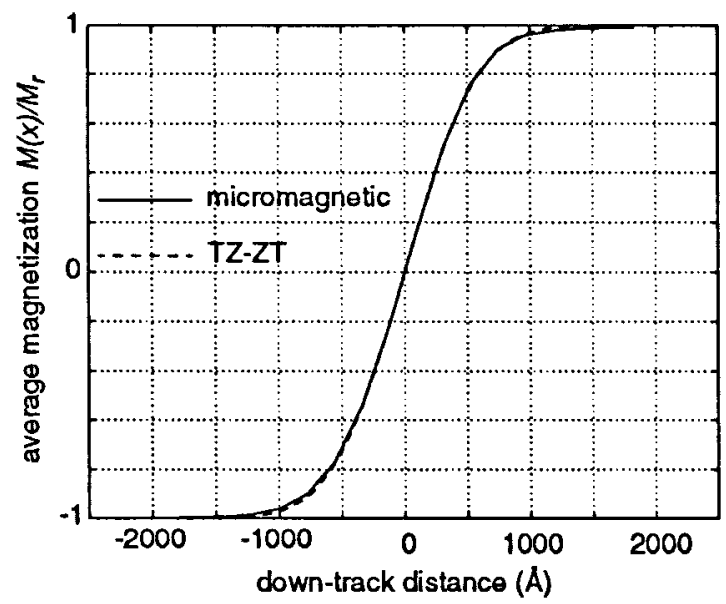

Fig. 7. Average transition profiles for the oriented medium. Solid line: micromagnetic model. Dotted line: TZ-ZT model.

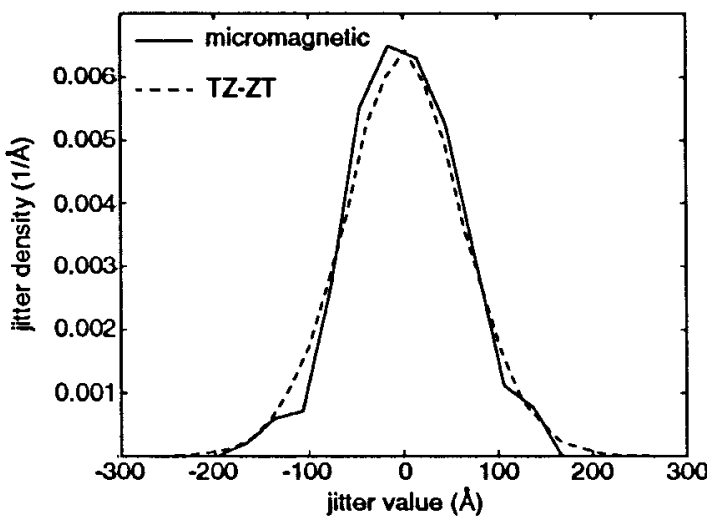

Fig. 8. Normalized jitter histograms (empirical pdf's) for the oriented medium. Solid line: micromagnetic model. Dotted line: TZ-ZT model.

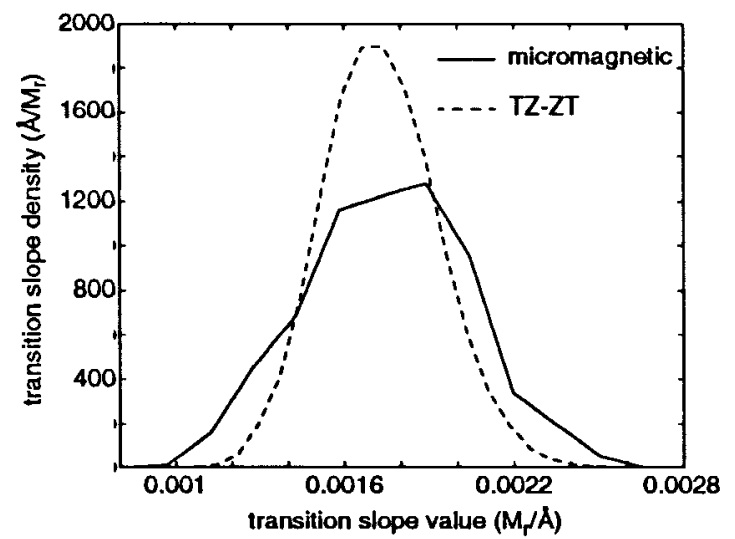

Fig. 9. Normalized transition slope histograms (empirical pdf's) for the oriented medium. Solid line: micromagnetic model. Dotted line: TZ-ZT model.

$\delta=250 \AA ; H_{c}=1700$ Oe; $M_{r}=600 \mathrm{emu} / \mathrm{cm}^{3}$. This was achieved at the expense of increasing the saturation magnetization of the isotropic medium by about $16 \%$ with respect to the oriented medium.

Following the same procedure as for the isotropic medium, we found the average magnetization profile for the isotropic

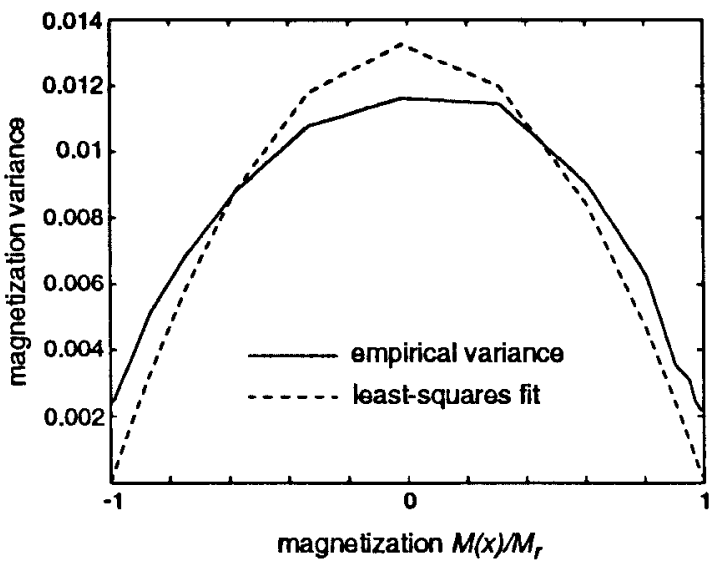

Fig. 10. Magnetization variance for the isotropic medium. Solid line: empirical magnetization variance $\hat{\sigma}_{M}(x)$ versus the magnetization $M(x) / M_{r}$. Dotted line: Least-squares parabola fit.

medium to be

$$
\frac{M(x)}{M_{r}} \approx M_{a}(x)=\operatorname{erf}\left(\frac{x}{\sigma \sqrt{2}}\right)
$$

with $\sigma=530 \AA$. The triangle heights pdf is then

$$
f_{H}(h)=-\frac{M_{a}^{\prime \prime}(h)}{M_{a}^{\prime}(0)}= \begin{cases}\frac{h}{\sigma^{2}} \exp \left(-\frac{h^{2}}{2 \sigma^{2}}\right) & h \geq 0 \\ 0 & h<0 .\end{cases}
$$

Using the least-squares parabola-fitting procedure, we find the cross-track correlation width to be

$$
s=165.76 \AA \text {. }
$$

Fig. 10 shows the plots of the empirical magnetization variance (solid line) and the parabola fit (dotted line) for the isotropic medium. The vertex angle obtained with (24) and (25) is

$$
\theta=2 a \tan \left[\frac{s \cdot \mathrm{E}[H]}{2 \cdot \operatorname{Var}(H)}\right] \approx 49^{\circ} .
$$

With the TZ-ZT defining quantities given in (24) and (26), we created 50000 independent transitions using the TZ-ZT model and compared their statistics to the micromagnetic transitions. The average magnetization profile comparison is shown in Fig. 11. The normalized jitter histograms are compared in Fig. 12. As in the case of the oriented medium, we see that, for the isotropic medium, the TZ-ZT model shows very good agreement for the average transition profile and jitter noise but underestimates the transition slope variance; see Fig. 13.

\section{CONCLUSION}

In this paper, we provided analytic tools for extracting the defining quantities for stochastic zig-zag transition noise models in longitudinal magnetic recording. The particular model we used was the TZ-ZT model. We derived a relationship linking the pdf of the triangle heights to an average transition profile. We determined the zig-zag angle by matching the cross-track correlation width of the TZ-ZT model to the crosstrack correlation width of the real media. We also provide an 


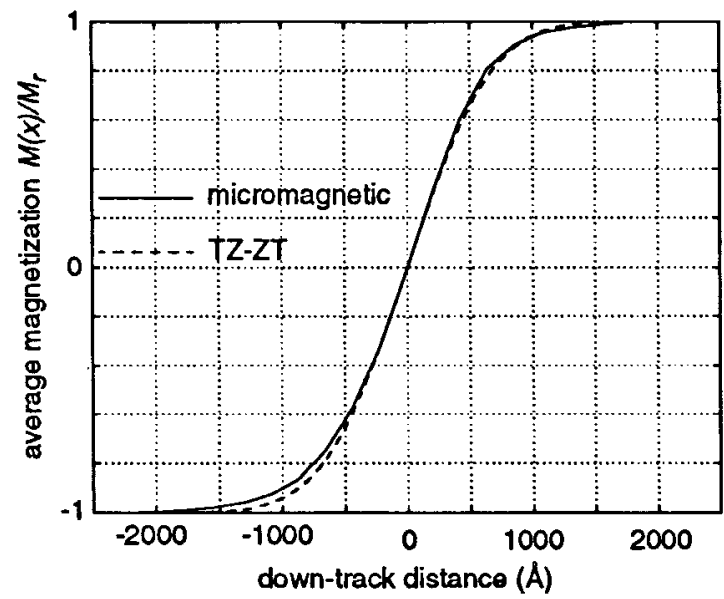

Fig. 11. Average transition profiles for the isotropic medium. Solid line: micromagnetic model. Dotted line: TZ-ZT model.

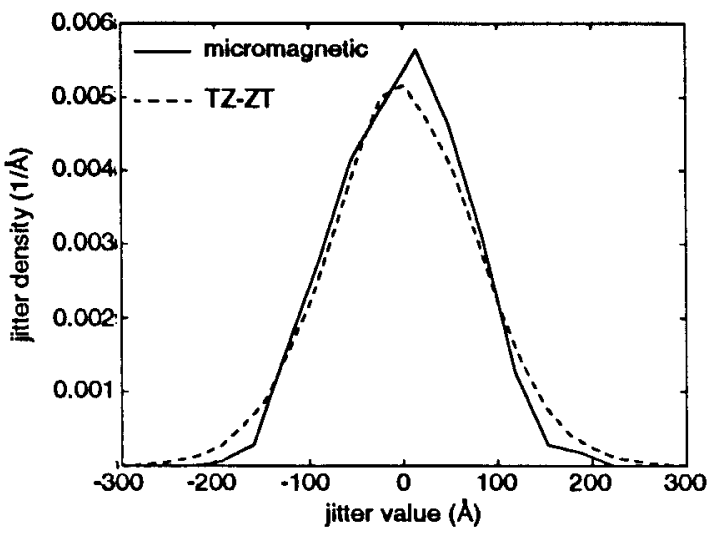

Fig. 12. Normalized jitter histograms (empirical pdf's) for the isotropic medium. Solid line: micromagnetic model. Dotted line: TZ-ZT model.

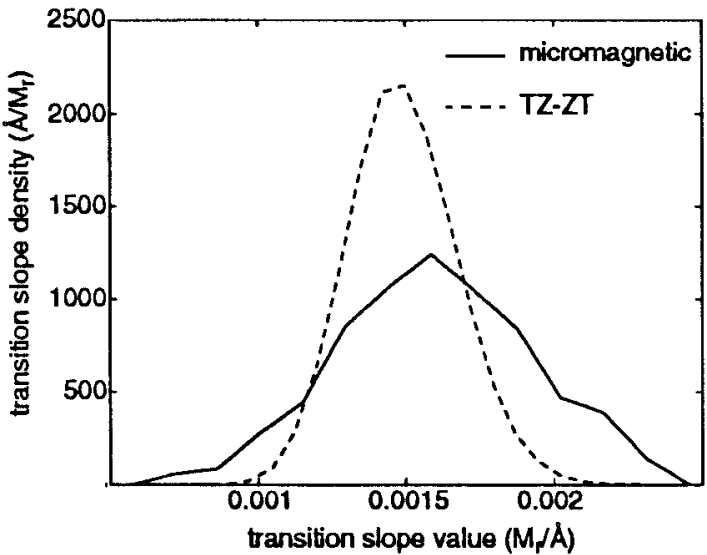

Fig. 13. Normalized transition slope histograms (empirical pdf's) for the isotropic medium. Solid line: micromagnetic model. Dotted line: TZ-ZT model.

alternative interpretation of the cross-track correlation width, linking this quantity to the statistics (mean and variance) of the magnetization cluster sizes. Through two examples (one for oriented media and one for isotropic media), we demonstrated how these results can be used in statistical media noise modeling.

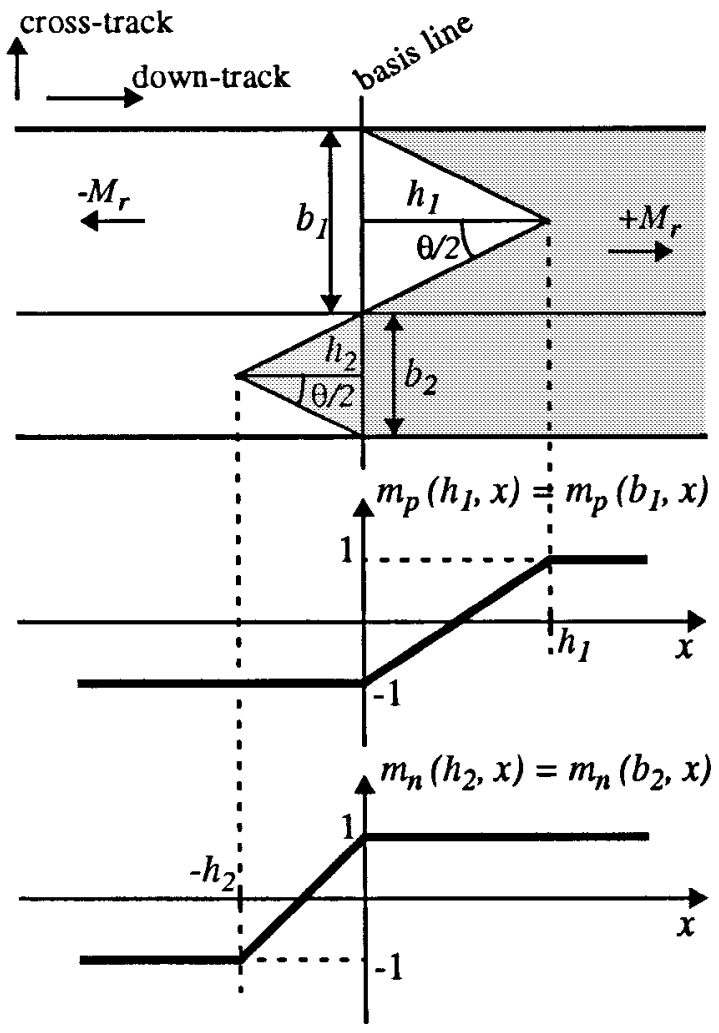

Fig. 14. Top: portion of a track involving only two triangles. Middle: magnetization profile in the stripe associated with the positive oriented triangle. Bottom: magnetization profile in the stripe associated with the negative oriented triangle.

With the analytic tools that we have presented, the stochastic zig-zag transition models (particularly the TZ-ZT model) have been made to be much easier to use. Although not explicitly discussed in this paper, extensions of the model to incorporate nonlinear effects such as nonlinear transition shift and percolation effects are also relatively easy to incorporate; see [19] and [24] for details. The stochastic zig-zag transition models are a much faster alternative to micromagnetic modeling (for example, TZ-ZT modeling is approximately four orders of magnitude faster than micromagnetic modeling). This makes them very attractive in statistical studies like error rate predictions, where thousands of statistically independent transitions need to be created in a short time period.

\section{APPENDIX A}

PROOFS OF RELATIONSHIPS BETWEEN MAGNETIZATION

Characteristics and Zig-Zag Transition Statistics

Proof of Theorem 1: Consider two adjacent triangles of opposite orientation, as in Fig. 14. The quantities $b_{1}$ and $h_{1}$ are the base and height of the positively oriented triangle, whereas $b_{2}$ and $h_{2}$ correspond to the negatively oriented triangle. Since the vertex angle $\theta$ is constant, $b_{i}=2 h_{i} \tan (\theta / 2)$, for $i$ $=1$, 2. For $h_{i}$ drawn from a pdf $f_{H}(h)$, it follows from this relationship that the pdf of the samples $b_{i}$ is $f_{B}(b)=$ $(1 / K) f_{H}(b / K)$, where $K=2 \tan (\theta / 2)$.

The magnetization profiles (normalized to $M_{r}$ ) in the stripes 
defined by $b_{1}$ and $b_{2}$ are

$$
\begin{aligned}
m_{p}\left(h_{1}, x\right) & =m_{p}\left(b_{1}, x\right) \\
& = \begin{cases}\frac{-1}{2} \\
\frac{2}{h_{1}}\left(x-\frac{h_{1}}{2}\right) & 0 \leq x \leq h_{1} \\
1 & x>h_{1}\end{cases} \\
m_{n}\left(h_{2}, x\right) & =m_{n}\left(b_{2}, x\right) \\
& = \begin{cases}\frac{-1}{2}\left(x+\frac{h_{2}}{2}\right) & -h_{2} \leq x \leq 0 \\
\frac{h_{2}}{1} & x>0 .\end{cases}
\end{aligned}
$$

The overall magnetization profile for an infinitely wide track is given by the average

$$
\begin{aligned}
M(x)= & \frac{1}{2} \int_{0}^{\infty} f_{\beta}\left(b_{1}\right) m_{p}\left(b_{1}, x\right) d b_{1} \\
& +\frac{1}{2} \int_{0}^{\infty} f_{\beta}\left(b_{2}\right) m_{n}\left(b_{2}, x\right) d b_{2}
\end{aligned}
$$

where $f_{\beta}\left(b_{1}\right)$ is the pdf of encountering a base $b_{1}$ of a positively oriented triangle and likewise for $f_{\beta}\left(b_{2}\right)$. The upfront factor of $\frac{1}{2}$ in (29) follows because the positively and negatively oriented triangles are equiprobable.

Note that $f_{\beta}(b) \neq f_{B}(b)$ since $f_{B}(b)$ is the pdf of the triangle bases, and $f_{\beta}(b)$ is the pdf of encountering a base of length $b$ by randomly choosing a point on the cross-track basis line. If we randomly pick a point on the basis line, we are more likely to fall on a base of a larger triangle than a smaller; hence, the two pdf's are different. Their exact relationship is

$$
f_{\beta}(b)=\frac{b \cdot f_{B}(b)}{\mathrm{E}[B]}
$$

where $\mathrm{E}[B]=\int_{0}^{\infty} b \cdot f_{B}(b) d b$ is the mean triangle base. Equation (30) is one of the main results describing the paradox of residual life in renewal theory; see, e.g., [21] and [22]. In earlier work [9], [10], this paradox was not recognized as it was erroneously assumed that $f_{\beta}(b)=f_{B}(b)$.

We manipulate (29) to derive (1). Because the triangle heights pdf $f_{H}(h)$ is the same for both positively and negatively oriented triangles, $M(x)$ is an odd function. Therefore, it suffices to consider only the case $x \geq 0$. Since the triangle bases are proportional to the triangle heights with the same constant of proportionality for all triangles, we have $\{[b$. $\left.\left.f_{B}(b)\right] / \mathrm{E}[B]\right\} \quad d b=\left\{\left[h \cdot f_{H}(h)\right] / \mathrm{E}[H]\right\} \quad d h$. Using this relationship and substituting (30) into (29), we obtain

$$
\begin{aligned}
M(x)= & \frac{1}{2} \int_{0}^{\infty} \frac{h_{1} \cdot f_{H}\left(h_{1}\right)}{\mathrm{E}[H]} m_{p}\left(h_{1}, x\right) d h_{1} \\
& +\frac{1}{2} \int_{0}^{\infty} \frac{h_{2} \cdot f_{H}\left(h_{2}\right)}{\mathrm{E}[H]} m_{n}\left(h_{2}, x\right) \mathrm{d} h_{1} \\
= & \frac{1}{2} \int_{0}^{\infty} \frac{h \cdot f_{H}(h)}{\mathrm{E}[H]}\left[m_{p}(h, x)+m_{n}(h, x)\right] d h .
\end{aligned}
$$

Notice that for $x \geq 0$

$$
\begin{aligned}
& m_{n}(h, x)=1 \text { and } \\
& m_{p}(h, x)= \begin{cases}\frac{2}{h}\left(x-\frac{h}{2}\right) & \text { for } h \geq x \\
1 & \text { for } h<x .\end{cases}
\end{aligned}
$$

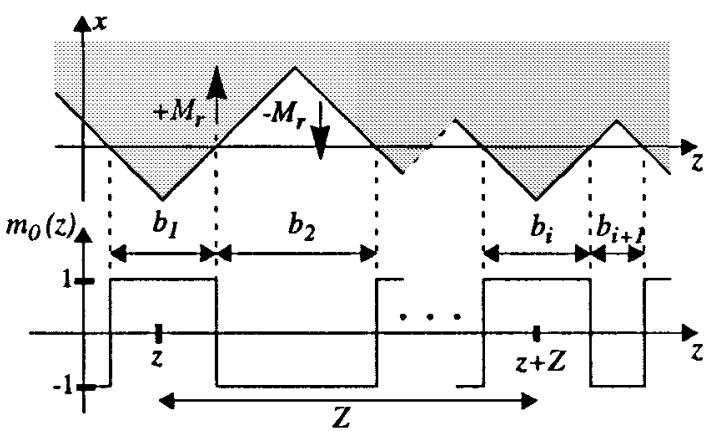

Fig. 15. Realization of an alternating renewal process $m_{0}(z)$.

Using (33), we rewrite (32) as

$$
\begin{aligned}
M(x)= & \frac{1}{2} \int_{0}^{x} \frac{h \cdot f_{H}(h)}{\mathrm{E}[H]} d h+\frac{1}{2} \int_{x}^{\infty} \frac{h \cdot f_{H}(h)}{\mathrm{E}[H]} \\
& \cdot \frac{2}{h}\left(x-\frac{h}{2}\right) d h+\frac{1}{2} \int_{0}^{\infty} \frac{h \cdot f_{H}(h)}{\mathrm{E}[H]} d h \\
= & \int_{0}^{x} \frac{h \cdot f_{H}(h)}{\mathrm{E}[H]} d h+x \int_{x}^{\infty} \frac{f_{H}(h)}{\mathrm{E}[H]} d h .
\end{aligned}
$$

The first derivative of (35) and its value at zero (the magnetization profile slope) are

$$
M^{\prime}(x)=\int_{x}^{\infty} \frac{f_{H}(h)}{\mathrm{E}[H]} d h \quad \text { and } \quad M^{\prime}(0)=\frac{1}{\mathrm{E}[H]}
$$

since $\int_{0}^{\infty} f_{H}(h) d h=1$. Similarly, we obtain the second derivative

$$
M^{\prime \prime}(x)=-\frac{f_{H}(x)}{\mathrm{E}[H]} .
$$

Substituting (36) into (37) and rearranging the equation, we get $f_{H}(x)=-\left[M^{\prime \prime}(x) / M^{\prime}(0)\right]$, for $x \geq 0$. Since triangle heights are nonnegative lengths, the pdf $f_{H}(h)$ has to be zero for negative arguments $h$. Therefore, the last relationship leads directly to (1).

Q.E.D.

Proof of Theorem 2: Assume, without loss of generality, that $M_{r}=1$. Let $m_{0}(z)=m(0, z)$. The function $m_{0}(z)$ is a random renewal process [21] that takes values of either -1 or 1 , whose realization is depicted in Fig. 15. The lengths between points where $m_{0}(z)$ changes sign are the "interarrival times" (interswitching intervals). They are equal to triangle bases in the TZ-ZT model because the basis line $x=0$ is where the zig-zag triangles are placed. If a random triangle height is $H$, then the corresponding random triangle base is $B=$ $2 H \tan (\theta / 2)$. Since in the TZ-ZT model the triangle heights are independent random variables, the interswitching intervals (the triangle bases) $B$ are also independent. Furthermore, the interswitching intervals $B$ are identically distributed with a pdf $f_{B}(b)=(1 / K) f_{H}(b / K)$, where $K=2 \tan (\theta / 2)$, and $f_{H}(h)$ is the pdf of the triangle heights.

According to (5), the cross-track correlation width $s_{\text {TZZZT }}$ is the integral of the cross-track autocorrelation function $r_{\mathrm{TZ}-\mathrm{ZT}}(Z)$, which is the autocorrelation of the cross-track magnetization $m_{0}(z)$, i.e., $r_{\mathrm{TZ}-Z \mathrm{~T}}(Z)=\mathrm{E}\left[m_{0}(z) m_{0}(z+Z)\right]$. Define $\mathcal{R}_{\mathrm{TZ}-\mathrm{ZT}}(f)=\int_{Z=-\infty}^{\infty} r_{\mathrm{TZ}-\mathrm{ZT}}(Z) e^{-j 2 \pi f Z} d Z$ as the Fourier transform of $r_{\mathrm{T} Z-Z \mathrm{~T}}(Z)$. From the properties of the 
Fourier transform, we have $s_{\mathrm{TZ}-\mathrm{ZT}}=\mathcal{R}_{\mathrm{TZ}-\mathrm{ZT}}(0)$. We will use this to prove Theorem 2 .

The cross track correlation function is equal to (we are assuming $M_{r}=1$ )

$$
r_{\mathrm{TZ}-\mathrm{ZT}}(Z)=\mathrm{E}\left[m_{0}(z) m_{0}(z+Z)\right]=\pi_{e}(Z)-\pi_{o}(Z) .
$$

Here, $\pi_{e}(Z)$ is the probability that an even number of switches occurs between the points $z$ and $z+Z$ for any $z$. Similarly, $\pi_{o}(Z)$ is the probability of an odd number of switches occurring in the same interval. While a series expansion of $r_{\mathrm{TZ}-\mathrm{ZT}}(Z)=\pi_{e}(Z)-\pi_{o}(Z)$ is given in [11], we take a different approach here. Define the causal Laplace transform of $g(t)$ as $G(s)=\int_{t=0}^{\infty} g(t) e^{-s t} d t$. The alternating renewal processes theory [21] gives the causal Laplace transforms $\Pi_{e}(s)$ and $\Pi_{o}(s)$ of $\pi_{e}(Z)$ and $\pi_{o}(Z)$

$$
\begin{aligned}
& \Pi_{e}(s)=\int_{Z=0}^{\infty} \pi_{e}(Z) e^{-s Z} \mathrm{~d} Z=\frac{1}{s}-\frac{1}{s^{2} \mathrm{E}(B)} \cdot \frac{1-F_{B}(s)}{1+F_{B}(s)} \\
& \Pi_{o}(s)=\int_{Z=0}^{\infty} \pi_{o}(Z) e^{-s Z} \mathrm{~d} Z=\frac{1}{s^{2} \mathrm{E}(B)} \cdot \frac{1-F_{B}(s)}{1+F_{B}(s)} .
\end{aligned}
$$

Here, $F_{B}(s)$ is the causal Laplace transform of the pdf $f_{B}(b)$. The causal Laplace transform of $r_{\mathrm{TZ}-\mathrm{ZT}}(Z)$ is then, according to $(38)$,

$$
R_{\mathrm{TZ}-\mathrm{ZT}}(s)=\Pi_{e}(s)-\Pi_{o}(s)=\frac{1}{s}-\frac{2}{s^{2} \mathrm{E}(B)} \cdot \frac{1-F_{B}(s)}{1+F_{B}(s)} .
$$

Since $r_{\mathrm{TZ}-\mathrm{ZT}}(Z)$ is an even function (it is an autocorrelation function), we have a relationship linking the Fourier transform $\mathcal{R}_{\mathrm{TZ}-\mathrm{ZT}}(f)$ to the causal Laplace transform $R_{\mathrm{TZ}-\mathrm{ZT}}(s)$

$$
\begin{aligned}
\mathcal{R}_{\mathrm{TZ}-\mathrm{ZT}}(f)= & \lim _{s \rightarrow j 2 \pi f}\left[R_{\mathrm{TZ}-\mathrm{ZT}}(s)+R_{\mathrm{TZ}-\mathrm{ZT}}(-s)\right] \\
= & \frac{2}{(2 \pi f)^{2} \mathrm{E}(B)} \\
& {\left[\frac{1-F_{B}(j 2 \pi f)}{1+F_{B}(j 2 \pi f)}+\frac{1-F_{B}(-j 2 \pi f)}{1+F_{B}(-j 2 \pi f)}\right] . }
\end{aligned}
$$

Here, $F_{B}(j 2 \pi f)$ is the causal Laplace transform of $f_{B}(b)$ evaluated at $s=j 2 \pi f$. Since $f_{B}(b)=0$ for $b<0$, we have that the Fourier transform of $f_{B}(b)$ equals $\mathcal{F}_{B}(f)=F_{B}(j 2 \pi f)$. Furthermore, because $f_{B}(b)$ is a pdf and thus a real function, we have $\mathcal{F}_{B}(-f)=\mathcal{F}_{B}^{*}(f)$, where the superscript * denotes complex conjugation. Thus, (43) takes its final form

$$
\mathcal{R}_{\mathrm{TZ}-\mathrm{ZT}}(f)=\frac{4}{(2 \pi f)^{2} \mathrm{E}(B)} \cdot \frac{1-\left|\mathcal{F}_{B}(f)\right|^{2}}{\left|1+\mathcal{F}_{B}(f)\right|^{2}} .
$$

We now prove (6) by taking the limit of (44) as $f \rightarrow 0$

$$
\begin{aligned}
s_{\mathrm{TZ}-\mathrm{ZT}} & =\mathcal{R}_{\mathrm{TZ}-\mathrm{ZT}}(0)=\lim _{f \rightarrow 0} \mathcal{R}_{\mathrm{TZ}-\mathrm{ZT}}(f) \\
& =\lim _{f \rightarrow 0} \frac{4}{(2 \pi f)^{2} \mathrm{E}(B)} \cdot \frac{1-\left|\mathcal{F}_{B}(f)\right|^{2}}{\left|1+\mathcal{F}_{B}(f)\right|^{2}} . \\
& =\frac{4}{\mathrm{E}(B)} \cdot \lim _{f \rightarrow 0} \frac{1}{\left|1+\mathcal{F}_{B}(f)\right|^{2}} \cdot \lim _{f \rightarrow 0} \frac{1-\left|\mathcal{F}_{B}(f)\right|^{2}}{(2 \pi f)^{2}} .
\end{aligned}
$$

Expand $\mathcal{F}_{B}(f)$ in a Taylor series

$$
\begin{aligned}
\mathcal{F}_{B}(f) & =\int_{b=-\infty}^{\infty} f_{B}(b) e^{-j 2 \pi f b} \mathrm{~d} b \\
& =\int_{b=-\infty}^{\infty}\left(\sum_{k=0}^{\infty} \frac{(-j 2 \pi f b)^{k}}{k !}\right) f_{B}(b) \mathrm{d} b \\
& =\sum_{k=0}^{\infty} \frac{(-j 2 \pi f)^{k}}{k !} \mathrm{E}\left(B^{k}\right) .
\end{aligned}
$$

Then, we have

$$
\begin{aligned}
\frac{1-\left|\mathcal{F}_{B}(f)\right|^{2}}{(2 \pi f)^{2}} & \\
= & \frac{1-\sum_{l=0}^{\infty} \sum_{k=0}^{\infty} \frac{(-j 2 \pi f)^{l}}{l !} \frac{(j 2 \pi f)^{k}}{k !} \mathrm{E}\left(B^{l}\right) \mathrm{E}\left(B^{k}\right)}{(j 2 \pi f)^{2}} \\
= & C_{1}+\sum_{n=2}^{\infty} C_{n}(2 \pi f)^{2(n-1)} .
\end{aligned}
$$

It is easy to verify that the first coefficient is $C_{1}=\mathrm{E}\left(B^{2}\right)-$ $\mathrm{E}(B)^{2}=\operatorname{Var}(B)$. Provided that the moments $\mathrm{E}\left(B^{n}\right)$ exist for all $n \geq 1$ (which is satisfied by most pdf's, e.g., Gaussian, Rayleigh, exponential, etc., with rare exceptions, e.g., the Cauchy pdf), we then have one of the limits in (46)

$$
\lim _{f \rightarrow 0} \frac{1-\left|\mathcal{F}_{B}(f)\right|^{2}}{(2 \pi f)^{2}}=C_{1}=\operatorname{Var}(B) .
$$

By straightforward substitution of $f=0$, we get the other limit in (46)

$$
\lim _{f \rightarrow 0} \frac{1}{\left|1+\mathcal{F}_{B}(f)\right|^{2}}=\frac{1}{\left|1+\mathcal{F}_{B}(0)\right|^{2}}=\frac{1}{|1+1|^{2}}=\frac{1}{4} .
$$

Notice that $\mathcal{F}_{B}(0)=\int_{b=-\infty}^{\infty} f_{B}(b) \mathrm{d} b=1$ since $f_{B}(b)$ is a pdf. Substituting (49) and (50) into (46), we get (6). Q.E.D.

\section{REFERENCES}

[1] R. A. Baugh, E. S. Murdock, and B. R. Natarajan, "Measurement of noise in magnetic media," IEEE Trans. Magn., vol. MAG-19, pp. 1722-1724, Sept. 1983.

[2] R. N. Belk, K. P. George, and S. G. Mowry, "Noise in high performance thin-film longitudinal magnetic recording media," IEEE Trans. Magn., vol. MAG-21, pp. 1350-1355, Sept. 1985.

[3] M. J. Freiser, "On the zigzag form of charged domain walls," IBM J. Res. Dev., vol. 23, pp. 330-338, May 1979.

[4] M. Muller and E. Murdock, "Williams-Comstock type model for sawtooth transitions in thin film media," IEEE Trans. Magn., vol. 23, pp. 2368-2370, Sept. 1987.

[5] X. Y. Zhang, H. Suhl, and P. K. George, "Relationship between the transition width and the zigzag wavelength," J. Appl. Phys., vol. 63, pp. 3257-3259, Apr. 1988.

[6] B. K. Middleton, J. J. Miles, and R. H. Noyau, "The digital recording properties of thin film media having sawtooth magnetization transitions," IEEE Trans. Magn., vol. 24, pp. 3099-3101, Nov. 1988.

[7] T. C. Arnoldussen and H. C. Tong, "Zigzag transition profiles, noise, and correlation statistics in highly oriented longitudinal media," IEEE Trans. Magn., vol. MAG-22, pp. 889-891, Sept. 1986.

[8] Y.-S. Tang and L. Osse, "Zig-zag domains and metal film disk noise," IEEE Trans. Magn., vol. MAG-23, pp. 2371-2373, Sept. 1987.

[9] B. K. Middleton and J. J. Miles, "Sawtooth magnetization transitions and the digital recording properties of thin film recording media," in Proc. IEE Conf., Apr. 1991, pp. 20-25.

[10] _ _ "Recorded magnetization distributions in thin film media," IEEE Trans. Magn., vol. 27, pp. 4954-4959, Nov. 1991. 
[11] A. Kavčić and J. M. Moura, "Triangle zig-zag transition modeling," Tech. Rep., Data Storage Syst. Center, Dept. Elect. Comput. Eng., Carnegie Mellon Univ., Pittsburgh, PA, 1995.

[12] J.-G. Zhu and N. H. Bertram, "Micromagnetic studies of thin metallic films," J. Appl. Phys., vol. 63, pp. 3248-3253, Apr. 1988.

[13] _ "Recording and transition noise simulations in htin film media," IEEE Trans. Magn., vol. 24, pp. 2706-2708, Nov. 1988.

[14] P.-L. Lu and S. Charap, "Angular variations of coercivity in thinfilm media by micromagnetic model," IEEE Trans. Magn., vol. 28, pp. 986-989, Mar. 1992.

[15] H. N. Bertram, Theory of Magnetic Recording. Cambridge, U.K.: Cambridge Univ. Press, 1994.

[16] J. Caroselli and J. K. Wolf, "A new model for media noise in thin film magnetic recording media," in Proc. SPIE: Coding Signal Process. Inform. Storage, vol. 2605, pp. 29-38, Oct. 1995.

[17] A. Kavčí and J. M. F. Moura, "Expedient media noise modeling: Isolated and interacting transitions," IEEE Trans. Magn., vol. 32, pp. 3875-3877, Sept. 1996.

[18] R. D. Brandt, A. J. Armstrong, H. N. Bertram, and J. K. Wolf, "A simple statistical model of partial erasure in thin film disk recording systems," IEEE Trans. Magn., vol. 27, pp. 4978-4980, Nov. 1991.

[19] A. Kavčić and J. M. F. Moura, "Experimental validation of the triangle zig-zag transition model," IEEE Trans. Magn., vol. 33, pp. 2704-2706, Sept. 1997.

[20] A. Papoulis, Probability, Random Variables and Stochastic Processes. New York: McGraw-Hill, 1965.

[21] D. R. Cox, Renewal Theory. London, U.K.: Methuen, 1962.

[22] L. Kleinrock, Queueing Systems. New York: Wiley, 1975.

[23] H. N. Bertram and X. Che, "General analysis of noise in recorded transitions in thin film recording media," IEEE Trans. Magn., vol. 29, pp. 201-208, 1993.

[24] A. Kavčić and J. M. F. Moura, "Signal generation model for high density magnetic recording," in Proc. IEEE GLOBECOM, London, U.K., Nov. 1996.

Aleksandar Kavčić (S'93) was born in Belgrade, Yugoslavia, in 1968. He received the Dipl.-Ing. degree in elecrical engineering from Ruhr University Bochum, Germany, in 1993 and has since been with Carnegie Mellon University, Pittsburgh, PA, where he is a Ph.D. candidate in the Department of Electrical and Computer Engineering.

His research interests are in signal processing, communications, and magnetic recording, while his thesis research is on modeling and detection in high-density recording.
José M. F. Moura (S'71-M'75-SM'90-F'94) received the engenheiro electrotécnico degree in 1969 from Instituto Superior Técnico (IST), Lisbon, Portugal, and the M.Sc., E.E., and the D.Sc. degrees in electrical engineering and computer science from the Massachusetts Institute of Technology (MIT), Cambridge, in 1973 and 1975, respectively.

$\mathrm{He}$ is presently a Professor of Electrical and Computer Engineering at Carnegie Mellon University (CMU), Pittsburgh, PA, which he joined in 1986. Prior to this, he was on the faculty of IST where he was an Assistant Professor (1975), Professor Agregado (1978), and Professor Catedrático (1979). He has had visiting appointments at several institutions, including MIT (Genrad Associate Professor of Electrical Engineering and Computer Science from 1984 to 1986) and the University of Southern California (research scholar, Department of Aerospace Engineering, Summers of 1978 to 1981). His research interests include statistical signal processing (one- and twodimensional), digital communications, image and video processing, detection and modeling in high density recording, and multiresolution techniques. $\mathrm{He}$ has organized and codirected two international scientific meetings on signal processing theory and applications. He has over 170 published technical contributions and is co-editor of two books.

Dr. Moura is currently the Editor-in-Chief for the IEEE TRANSACTIONS ON Signal Processing and a member of the Underwater Acoustics Technical Committee of the IEEE Signal Processing Society. He was a member of the IEEE Press Board (1991-1995), a Technical Associate Editor for the IEEE Signal Processing LetTers (1993-1995), and an Associate Editor for the IEEE Transactions on Signal Processing (1988-1992). He was a program committee member for the IEEE International Conference on Image Processing (ICIP'95) and for the IEEE International Symposium on Information Theory (ISIT'93). He is a corresponding member of the Academy of Sciences of Portugal (Section of Sciences). He is affiliated with several IEEE societies, Sigma Xi, AMS, IMS, and SIAM. 